\title{
802.11 LANs: Saturation Throughput in the Presence of Noise ${ }^{\star}$
}

\author{
Vladimir Vishnevsky and Andrey Lyakhov \\ Institute for Information Transmission Problems of RAS \\ B. Karetny 19, Moscow, 101447, Russia \\ \{vishn, lyakhov\}@iitp.ru \\ http://www.iitp.ru
}

\begin{abstract}
IEEE 802.11 specifies a technology for wireless local area networks (LANs) and mobile networking. In this paper, we present an analytical method of estimating the saturation throughput of 802.11 wireless LAN in the presence of noise which distorts transmitted frames. Besides the Basic Access mechanism of the 802.11 MAC protocol, we study such optional tool as the RTS/CTS method, which allows reducing the influence of collisions. In addition to the throughput, our method allows estimating a probability of a packet rejection occurring when the number of packet transmission retries attains its limit. The obtained numerical results of investigating 802.11 LANs by this method are validated by simulation and show high estimation accuracy for any values of protocol parameters and bit error rates. These results also show that the method is an effective tool for tuning the protocol parameters.
\end{abstract}

\section{Introduction}

In recent years, wireless data communications networks have become one of the major trends of the network industry development. Wireless LANs can be considered as an extension of the wired network with a wireless "last mile" link for connecting a large number of mobile terminals. The obvious merit of wireless LANs is the simplicity of implementation-no cables are required, its topology can be dynamically changed with connection, movement, and disconnection of mobile users without much loss of time.

The success of wireless networks depends largely on the development of networking products for multiple access to a wireless medium and of the appropriate standards. One of such standards is the IEEE 802.11 protocol [1] concerning the specifications on MAC and PHY layers for wireless networks. Leading companies (e.g., CISCO) have developed software and hardware in conformity with this standard.

The fundamental access mechanism in the IEEE 802.11 protocol is the Distributed Coordination Function (DCF), which implements the Carrier Sense

\footnotetext{
* This work was partially supported by NATO Science Programme in the Collaborative Linkage Grant PST.CLG.977405 "Wireless Access to Internet exploiting the IEEE 802.11 technology"
}

E. Gregori et al. (Eds.): NETWORKING 2002, LNCS 2345, pp. 1008-1019 2002.

(C) Springer-Verlag Berlin Heidelberg 2002 
Multiple Access with Collision Avoidance (CSMA/CA) method. In this method, sequential attempts to transfer by every station are separated by backoff intervals. The number of slots $b$ in this interval is random and defined by a binary exponential backoff rule (see Section 2).

In previous works, performance of the DCF has been evaluated either by simulation (e.g., 2] ) or by approximate analytical models [3.4 based on assumptions simplifying considerably the backoff rule. The DCF scheme has been studied in depth in [5]-7], in which analytical methods have been developed for evaluating the performance of 802.11 wireless LANs in the saturation conditions when there are always queues for transmitting at every wireless LAN station. This performance index called the saturation throughput in [5] has been evaluated in the assumption of ideal channel conditions, i.e., in the absence of noise and hidden stations. The assumption of the absence of hidden stations is admissible as a result of the small distance between LAN stations. But if noise is neglected, the throughput may be overestimated, because electromagnetic noise in large cities is inevitable and worsens the throughput due to data distortion. In this paper we develop methods [5]-7] to study the influence of noise on the 802.11 LAN performance.

Further in Section 2 we briefly review the DCF operation in saturation and noise. In Sections 3 and 4 we develop a new analytical method of estimating the saturation throughput and a probability of a packet rejection occurring when the number of transmission retries attains its limit. In section 5, we give some numerical research results of the saturation throughput of 802.11 LANs. These results obtained by both our analytical method and simulation allow us to validate the developed method. Finally, the obtained results are summarized in section 6 .

\section{DCF in Saturation}

Now we briefly outline the DCF scheme, considering only the aspects that are exhibited in saturation and with absence of hidden stations. This scheme is described in detail in [1].

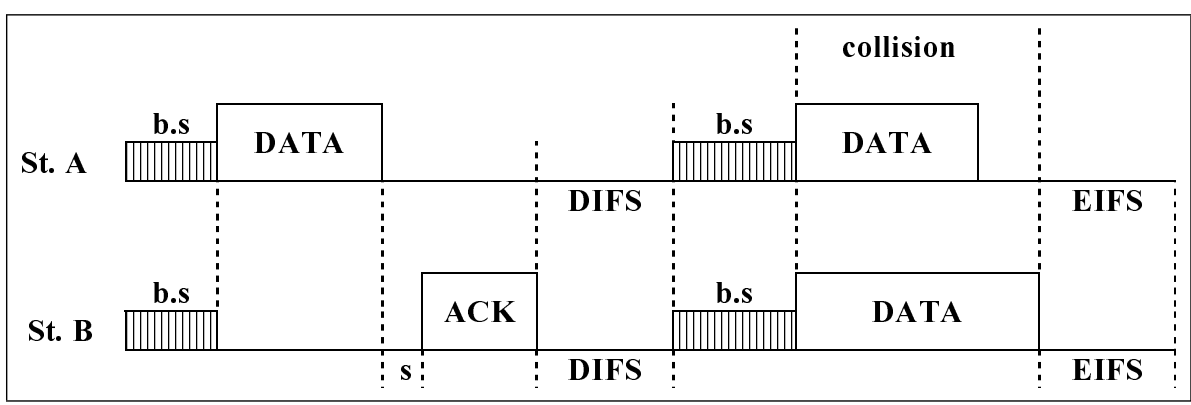

Fig. 1. Basic Access Mechanism (s - SIFS, b.s - backoff slots) 
Under the DCF, data packets are transferred in general via two methods. Short packets of length not greater than $\bar{P}$ are transferred by the Basic Access mechanism. In this mechanism shown in Figure 1, a station confirms the successful reception of a DATA frame by a positive acknowledgment ACK after a short SIFS interval.

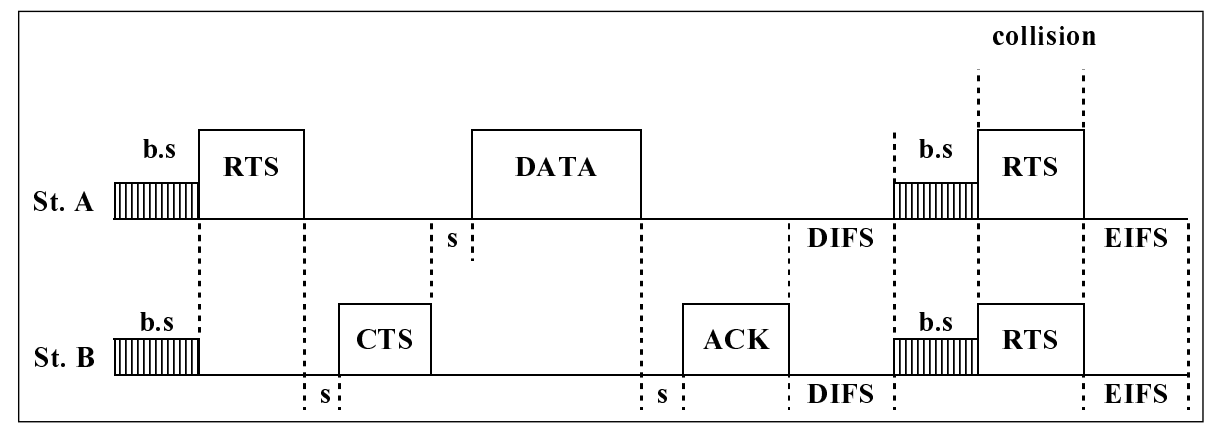

Fig. 2. RTS/CTS mechanism

Packets of length greater than the limit $\bar{P}$ called the RTS threshold in [1] are transferred via the Request-To-Send/Clear-To-Send (RTS/CTS) mechanism. In this case shown in Figure 2 first an inquiring RTS frame is sent to the receiver station, which replies by a CTS frame after a SIFS. Then only a DATA frame is transmitted and its successful reception is confirmed by an ACK frame. Since there are no hidden stations in the considered LAN, all other stations hear the RTS frame transmission and defer from their own attempts. This protects CTS, DATA and ACK frames from a collision-induced distortion. The RTS threshold $\bar{P}$ is chosen as a result of a reasonable trade-off between the RTS/CTS mechanism overhead consisting in transmitting two additional control frames (RTS and CTS) and reduction of collision duration. Figures 1 and 2 show that the collision duration is determined by the length of the longest packet involved in collision for the Basic Access mechanism, whereas in the RTS/CTS mechanism it is equal to the time of transferring a short RTS frame.

After a packet transfer attempt the station passes to the backoff state after a DIFS interval if the attempt was successful (i.e., there was no collision, all frames of a packet were transferred without noise-induced distortions) or after an EIFS interval if the attempt failed. The backoff counter is reset to the initial value $b$, which is called the backoff time, measured in units of backoff slots of duration $\sigma$, and chosen uniformly from a set $(0, \ldots, w-1)$. The value $w$, called the contention window, depends on the number $n_{r}$ of attempts performed for transmitting the current packet: $w=W_{n_{r}}$, where

$$
W_{n_{r}}=W_{0} 2^{n_{r}} \text { for } n_{r} \leq m \text { and } W_{n_{r}}=W_{m} \text { for } n_{r}>m,
$$

i.e., $w$ is equal to the minimum $W_{0}$ before the first attempt, then $w$ is doubled after every failed attempt of the current packet transmission, reaching the 
maximum $W_{m}=W_{0} 2^{m}$. Note that every transmission attempt of a packet can include transfers of several frames (RTS, CTS, DATA, and ACK). Backoff interval is reckoned only as long as the channel is free: the backoff counter is decreased by one only if the channel was free in the whole previous slot. Counting the backoff slots stops when the channel becomes busy, and backoff time counters of all stations can decrement next time only when the channel is sensed idle for the duration of $\sigma+$ DIFS or $\sigma+$ EIFS if the last sensed transmission is successful or failed, respectively. When the backoff counter attains its zero value, the station starts transmission.

In the course of transmission of a packet, a source station counts the numbers of short $\left(n_{s}\right)$ and long $\left(n_{\ell}\right)$ retries. Let a source station transfer a DATA frame with a packet of length equal to or less than $\bar{P}$, or an RTS frame. (Retries for these frames are called short ones in [1]). If a correct ACK or CTS frame, respectively, is received within timeout, then the $n_{s}$-counter is zeroed; otherwise $n_{s}$ is advanced by one. Similarly, the $n_{\ell}$-counter is zeroed or advanced by one in case of reception or absence of a correct ACK frame (within timeout) confirming the successful transfer of a DATA frame with a packet of length greater than $\bar{P}$ (transfer retries for that sort of DATA frames are called long retries). When any of these counters $n_{s}$ and $n_{\ell}$ attains its limit $N_{s}$ or $N_{\ell}$ respectively, the current packet is rejected. After the rejection or success of a packet transmission the next packet is chosen (due to saturation) with zeroing the values of $n_{r}, n_{s}$, and $n_{\ell}$.

As in [56], to study the DCF, we adopt the following assumption: all stations change their backoff counter after a DIFS or EIFS interval closing a packet transmission attempt, i.e., the source station (or stations in case of collision), which has performed a transmission, modifies its contention window $w$ and chooses randomly the backoff counter value from the set $(0, \ldots, w-1)$, while other stations just decrease their backoff counters by 1 (in reality [1], other stations can do it only after a backoff slot $\sigma$ since the end of the DIFS or EIFS interval). Thus, at the beginning of each slot any station can start its transmission. As shown in [7], this assumption does not affect significantly the throughput estimation results with the $W_{0}$ values recommended in [1].

\section{Throughput Evaluation}

Let us consider a wireless LAN of $N$ statistically homogeneous stations working in saturation. In fact, we mean by $N$ not a number of all stations of the LAN, but a number of active stations whose queues are not empty for a quite long observation interval. By statistically homogeneity of stations, we mean that the lengths of packets chosen by every station from the queue have identical probability distribution $\left\{d_{\ell}, \quad \ell=\ell_{\min }, \ldots, \ell_{\max }\right\}$. Since the distance between stations is small, we assume that there are no hidden stations and noise occurs concurrently at all stations. These assumptions imply that all stations "sense" the common wireless channel identically.

As in [5], let us subdivide the time of the LAN operation into non-uniform virtual slots such that every station changes its backoff counter at the start of a virtual slot and can begin transmission if the value of the counter becomes zero. 
Such a virtual slot is either (a) an "empty" slot in which no station transmits, or (b) a "successful" slot in which one and only one station transmits, or (c) a "collisional" slot in which two or more stations transmit.

As in 56], we assume that the probability that a station starts transmitting a packet in a given slot depends neither on the previous history, nor on the behavior of other stations, and is equal to $\tau$, which is the same for all stations. Hence the probabilities that an arbitrarily chosen virtual slot is "empty" $\left(p_{e}\right)$, "successful" $\left(p_{s}\right)$, or "collisional" $\left(p_{c}\right)$ are

$$
p_{e}=(1-\tau)^{N}, \quad p_{s}=N \tau(1-\tau)^{N-1}, \quad p_{c}=1-p_{e}-p_{s} .
$$

Thus, the throughput $S$ is determined by the formula

$$
S=\frac{p_{s} U}{p_{e} \sigma+p_{s} T_{s}+p_{c} T_{c}},
$$

where $T_{s}$ and $T_{c}$ are the mean duration of "successful" and "collisional" slots, respectively, and $U$ is the mean number of successfully transferred data bytes in a "successful" slot.

The duration of a "collisional" slot is the sum of time of transmitting the longest frame involved in collision and an EIFS interval. Disregarding the probability of collision of three or more frames, we obtain the formula for the mean duration of a "collisional" slot

$$
\begin{gathered}
T_{c}=\sum_{\ell=\ell_{\min }}^{\bar{P}} t_{d}(\ell) \widehat{d}_{\ell}\left\{\widehat{d}_{\ell}+2\left(\sum_{k=\ell_{\min }}^{\ell-1} \widehat{d}_{k}+\sum_{k=\bar{P}+1}^{\ell_{\max }} \widehat{d}_{k}\right)\right\}+t_{R T S}\left(\sum_{\ell=\bar{P}+1}^{\ell_{\max }} \widehat{d}_{\ell}\right)^{2} \\
+E I F S+\delta
\end{gathered}
$$

where $t_{d}(\ell)=H+\ell / V$ is the transmission time of a DATA frame including a packet of length $\ell$ and a header transmitted in time $H, V$ is the channel rate, $t_{R T S}$ is the transfer time for an RTS frame (according to [1], $t_{R T S}<H$ ), and $\delta$ is the propagation delay assumed the same for all pairs of stations. Finally, $\widehat{d}_{\ell}$ is the probability that the performed attempt is related to a packet of length $\ell$. Note that the distribution $\left\{\widehat{d}_{\ell}, \quad \ell=\ell_{\min }, \ldots, \ell_{\max }\right\}$ is different from the distribution $\left\{d_{\ell}, \quad \ell=\ell_{\min }, \ldots, \ell_{\max }\right\}$, because the longer the length of a packet, the greater the number of attempts required for transferring a packet due to the higher probability of distortion of the corresponding DATA frame by noise.

At the beginning of a "successful" slot, one and only one station initiates an attempt of transmitting a packet of length $\ell$, and this transmission is successful with probability $\pi_{h}(\ell)$ if none of the frames exchanged between the sender and receiver in this process is distorted by noise, i.e.,

$$
\pi_{h}(\ell)=\left[1-\xi_{d}(\ell)\right]\left(1-\xi_{a}\right) \quad \text { for } \quad \ell \leq \bar{P}
$$

and

$$
\pi_{h}(\ell)=\left(1-\xi_{r c}\right)\left[1-\xi_{d}(\ell)\right]\left(1-\xi_{a}\right) \quad \text { for } \quad \ell>\bar{P}
$$


where $\xi_{r c}=1-\left(1-\xi_{r c}\right)\left(1-\xi_{a}\right)$ is the probability of distorting an RTS-CTS sequence by noise, while $\xi_{d}(\ell), \xi_{r}$, and $\xi_{a}$ are the probabilities of noise-induced distortion of a DATA frame including a packet of length $\ell\left(\xi_{d}(\ell)\right)$, and RTS $\left(\xi_{r}\right)$ frame, and CTS and ACK $\left(\xi_{a}\right)$ frames of identical format [1]. These distortion probabilities are defined by the Bit Error Rate (BER) - the probability of distortion of a bit, i.e., an $\ell_{f}$-byte frame is distorted with probability $\xi_{\ell_{f}}=1-\exp \left\{-8 \ell_{f} \mathrm{BER}\right\}$. Transfer of a packet is terminated when an exchanged frame is distorted. Thus, the mean duration of a transfer attempt in a "successful" slot depends on the length $\ell$ of the transferred packet and is equal to

$$
\begin{aligned}
t_{s}(\ell)= & t_{d}(\ell)+\delta+\left[1-\xi_{d}(\ell)\right]\left(t_{A C K}+\operatorname{SIFS}+\delta\right) \\
& +\pi_{h}(\ell) \text { DIFS }+\left[1-\pi_{h}(\ell)\right] \text { EIFS }
\end{aligned}
$$

for $\ell \leq \bar{P}$ and

$$
\begin{gathered}
t_{s}(\ell)=t_{R T S}+\delta+\left(1-\xi_{r}\right)\left(t_{C T S}+\operatorname{SIFS}+\delta\right) \\
+\left(1-\xi_{r c}\right)\left\{\left[1-\xi_{d}(\ell)\right]\left(t_{A C K}+\operatorname{SIFS}+\delta\right)+t_{d}(\ell)+\operatorname{SIFS}+\delta\right\} \\
+\pi_{h}(\ell) \mathrm{DIFS}+\left[1-\pi_{h}(\ell)\right] \mathrm{EIFS}
\end{gathered}
$$

for $\ell>\bar{P}$, where $t_{C T S}=t_{A C K}$ is the transfer time of a CTS and an ACK frame.

Thus, the mean duration $T_{s}$ of a "successful" slot and the mean number of successfully transferred bytes $U$ in this slot are

$$
T_{s}=\sum_{\ell=\ell_{\min }}^{\ell_{\max }} t_{s}(\ell) \widehat{d}_{\ell}, \quad U=\sum_{\ell=\ell_{\min }}^{\ell_{\max }} \ell \pi_{h}(\ell) \widehat{d}_{\ell} .
$$

Therefore we have found all components of (3). So the throughput $S$ can be found if the transmission commencement probability $\tau$ and the probability distribution $\left\{\widehat{d}_{\ell}\right\}$ are known.

\section{Transmission Probability}

Now we study the process of transmitting a packet of length $\ell$ by some station. This process starts at the instance when the packet is chosen from the queue and ends with either this packet successful transmission or its rejection. Let $f_{\ell}$ and $\bar{w}_{\ell}$ be the mean numbers of this packet transmission attempts and virtual slots in which the considered station defers from transmission during this process. Then

$$
\begin{gathered}
\tau=\sum_{\ell=\ell_{\min }}^{\ell_{\max }} d_{\ell} f_{\ell} / \sum_{\ell=\ell_{\min }}^{\ell_{\max }} d_{\ell}\left(f_{\ell}+\bar{w}_{\ell}\right), \\
\widehat{d}_{\ell}=d_{\ell} f_{\ell} / \sum_{k=\ell_{\min }}^{\ell_{\max }} d_{k} f_{k}, \quad \ell=\overline{\ell_{\min }, \ell_{\max }} .
\end{gathered}
$$


Moreover, we will seek also the averaged probability $\bar{p}_{r e j}$ of packet rejection when one of the counters $n_{s}$ or $n_{\ell}$ attains its limiting value $N_{s}$ or $N_{\ell}$, respectively. This probability can be found from the following sum:

$$
\bar{p}_{r e j}=\sum_{\ell=\ell_{\min }}^{\ell_{\max }} d_{\ell} p_{r e j}(\ell),
$$

where $p_{r e j}(\ell)$ is the probability of rejecting a packet of length $\ell$.

In the course of transmitting a packet of length $\ell$ let exactly $i$ attempts take place. Let $\psi_{\ell}(i)$ denote the probability of this event. Obviously,

$$
\psi_{\ell}(i)=\psi_{\ell}^{s}(i)+\psi_{\ell}^{r}(i)
$$

where $\psi_{\ell}^{s}(i)$ and $\psi_{\ell}^{r}(i)$ are the probabilities that this transmission process terminates at attempt $i$ with success and rejection, respectively. In our case when exactly $i$ attempts take place, the mean number of virtual slots in which the station defers from transmission in the course of the whole considered process is

$$
\begin{gathered}
\bar{W}_{i}=\sum_{k=0}^{i-1} \frac{W_{k}-1}{2}=W_{i-1}-\frac{W_{0}+i}{2}, \quad 1 \leq i \leq m+1, \\
\bar{W}_{i}=\sum_{k=0}^{m} \frac{W_{k}-1}{2}+\frac{W_{m}-1}{2}(i-1-m)=W_{m} \frac{i-m+1}{2}-\frac{W_{0}+i}{2}, \quad i>m+1 .
\end{gathered}
$$

Then we have

$$
f_{\ell}=\sum_{i=1}^{i_{m}(\ell)} i \psi_{\ell}(i), \quad \bar{w}_{\ell}=\sum_{i=1}^{i_{m}(\ell)} \bar{W}_{i} \psi_{\ell}(i),
$$

where $i_{m}(\ell)$ is the maximal number of attempts for such a packet, i.e., $i_{m}(\ell)=N_{s}$ for $\ell \leq \bar{P}$ and $i_{m}(\ell)=i_{m}^{1}=\left(N_{s}-1\right) N_{\ell}+1$ for $\ell>\bar{P}$.

Now we look for probabilities $\psi_{\ell}(i)$. First we consider a simple case $\ell \leq$ $\bar{P}$, in which the number $i$ of attempts is bounded by $N_{s}$. The probability of unsuccessful attempt is

$$
\pi_{c d}(\ell)=1-\left(1-\pi_{c}\right)(1-\xi(\ell))
$$

where

$$
\pi_{c}=1-(1-\tau)^{N-1} \quad \text { and } \quad \xi(\ell)=1-\left(1-\xi_{d}(\ell)\right)\left(1-\xi_{a}\right) \varnothing
$$

are the probabilities of the current attempt collision and distorting DATA or ACK frames, respectively. Then the process is completed successfully at the $i$ th attempt with probability

$$
\psi_{\ell}^{s}(i)=\left[1-\pi_{c d}(\ell)\right]\left[\pi_{c d}(\ell)\right]^{i-1}, \quad i=1, \ldots, N_{s},
$$

or ends in rejection with probability

$$
p_{r e j}(\ell)=\left[\pi_{c d}(\ell)\right]^{N_{s}},
$$

i.e., 


$$
\psi_{\ell}^{r}(i)=0 \quad \text { for } \quad i<N_{s} \text { and } \quad \psi_{\ell}^{r}\left(N_{s}\right)=\left[\pi_{c d}(\ell)\right]^{N_{s}} .
$$

Consequently by (9),

$$
\psi_{\ell}(i)=\left[1-\pi_{c d}(\ell)\right]\left[\pi_{c d}(\ell)\right]^{i-1}, \quad i=1, \ldots, N_{s}-1, \quad \psi_{\ell}\left(N_{s}\right)=\left[\pi_{c d}(\ell)\right]^{N_{s}-1} .
$$

Now let $\ell>\bar{P}$. In this case the number $i_{d}$ of DATA frame transfer attempts is bounded by $N_{\ell}$ and each of these attempts may be preceded by $0, \ldots, N_{s}-1$ unsuccessful attempts of transferring an RTS frame. Moreover, in the case of a packet rejection due to attaining the limit $N_{s}$, the packet transmission process completes with $N_{s}$ failed RTS transfer attempts.

Let us express the probability $\psi_{\ell}^{r}(i)$ as the sum

$$
\psi_{\ell}^{r}(i)=p_{r e j}^{d}(\ell, i)+p_{r e j}^{r}(\ell, i)
$$

where $p_{r e j}^{d}(\ell, i)$ and $p_{r e j}^{r}(\ell, i)$ are the probabilities of rejection after $i$ packet transmission attempts due to the attainment of limiting values of the $n_{\ell^{-}}$and $n_{s}$-counters, respectively. Note that

$$
p_{r e j}(\ell)=\sum_{i=1}^{i_{m}^{1}}\left[p_{r e j}^{d}(\ell, i)+p_{r e j}^{r}(\ell, i)\right]
$$

The probabilities of unsuccessful transfer of DATA and RTS frames are $\xi(\ell)$ and $\pi_{c r}=1-\left(1-\pi_{c}\right)\left(1-\xi_{r c}\right)$, respectively. Therefore after simple algebraic operations we obtain

$$
\begin{gathered}
\psi_{\ell}^{s}(i)=\left(1-\pi_{c r}\right)[1-\xi(\ell)] \pi_{c r}^{i-1} \sum_{h=0}^{\min \left(i, N_{\ell}\right)-1}\left(\frac{\rho_{\ell}}{\pi_{c r}}\right)^{h} g(i-1-h, h+1), \quad i=1, \ldots, i_{m}^{1}, \\
p_{r e j}^{d}(\ell, i)=0, \quad i=1, \ldots, N_{\ell}-1, \\
p_{r e j}^{d}(\ell, i)=\pi_{c r}^{i-N_{\ell}} \rho_{\ell}^{N_{\ell}} g\left(i-N_{\ell}, N_{\ell}\right), \quad i=N_{\ell}, \ldots, i_{m}^{1}, \\
p_{r e j}^{r}(\ell, i)=0, \quad i=1, \ldots, N_{s}-1, \quad p_{r e j}^{r}\left(\ell, N_{s}\right)=\pi_{c r}^{N_{s}}, \\
p_{r e j}^{r}(\ell, i)=\pi_{c r}^{i} \min \left(i-N_{s}, N_{\ell}-1\right) \\
\sum_{h=1}\left(\frac{\rho_{\ell}}{\pi_{c r}}\right)^{h} g\left(i-N_{s}-h, h\right), \quad i=N_{s}+1, \ldots, i_{m}^{1},
\end{gathered}
$$

where $\rho_{\ell}=\left(1-\pi_{c r}\right) \xi(\ell)$ is the probability that an attempt of transmitting a packet of length $\ell$ fails just due to noise-induced distortion of DATA or ACK frames, while $g(u, v)$ is the number of ways in which $u$ indistinguishable balls (failed RTS transfer attempts) can be placed in $v$ urns (gaps preceding each of DATA transfers) so that every urn contains not more than $N_{s}-1$ balls. The function $g(u, v)$ is computed recursively:

$$
g(0, v)=1 \quad \forall v>0, \quad g(u, 1)=1 \text { for } u<N_{s} \text { and } 0 \text { for } u \geq N_{s}
$$




$$
g(u, v)=\sum_{k=0}^{\min \left(u, N_{s}-1\right)} g(u-k, v-1) \quad \text { for } \quad v \geq 2, \quad u>0 .
$$

Therefore the transmission probability $\tau$ can be estimated by the following iterative procedure.

Step 0. Define an initial value for $\tau$.

Step 1. For all possible packet lengths $\ell$ and number of attempts $i$, compute the rejection probabilities $\psi_{\ell}(i)$ by (14) if $\ell \leq \bar{P}$ or by (9), (15), and (17)-(19) if $\ell>\bar{P}$.

Step 2. For all possible packet lengths $\ell$, using (10), compute the mean numbers of attempts $f_{\ell}$ and virtual slots $\bar{w}_{\ell}$ in which transmission is postponed.

Step 3. Using (6), find the modified value of $\tau$ and compare it with the initial value. If the difference of these values is greater than a predefined limit, return to Step 1 using a new initial value for $\tau$ - the half-sum of its old initial value and the modified value.

After this iterative procedure, we obtain the averaged rejection probability $\bar{p}_{r e j}$ by (18), (12), (16), (18), and (19). Finally, we find the distribution $\left\{\widehat{d}_{\ell}\right\}$ by (7) and throughput by the formulas of the previous section.

We don't prove exactly the convergence of this iterative technique due to its complexity and lack of space. It is clear intuitively that the equation (6) has a unique solution because a growth of transmission probability $\tau$ leads to increasing the collision probability and, hence, to increasing the average number $\bar{w}_{\ell} / f_{\ell}$ of slots anticipating an attempt for all $\ell$. In practice, numerous examples of adopting the suggested technique with various values of wireless LAN parameters have shown that this technique provides very fast convergence to the solution and high speed of calculating the values of estimated performance indices. It takes less than a second to calculate $S$ and $\bar{p}_{\text {rej }}$ with running this technique program implementation at Intel Celeron $400 \mathrm{MHz}$.

\section{$5 \quad$ Numerical Results}

To validate our model, we have compared its results with that obtained by GPSS (General Purpose Simulation System) simulation 8]. The object of our numerical investigations was a LAN which consisted of $N$ statistically homogeneous stations working in saturation and was controlled by the DCF scheme of the IEEE 802.11 protocol with the higher-speed physical layer extension (802.11b) [9]. The values of protocol parameters used to obtain numerical results for the analytical model and simulation were the default values 9] for the Short Preamble mode and summarized in Table 1. Moreover, the information packet size $\ell$ (in bytes) is sampled uniformly from the set $\{1, \ldots, 1999\}$.

In our simulation model, we have tried to take into account of all real features of the 802.11 MAC protocol and, of course, not adopted the assumptions used with analytical modeling and described at the end of Section [2 and in Section [3] In the course of each run (it took about 2 hours, in average) of the simulation model, we watched the measured performance index value and stopped the simulation when this value fluctuations became quite small (within $0.5 \%$ ). 
Table 1. Values of protocol parameters

\begin{tabular}{|l|c||l|c|}
\hline Slot time, $\sigma$ & $20 \mu \mathrm{s}$ & Propagation time, $\delta$ & $1 \mu \mathrm{s}$ \\
\hline MAC+PHY Header & 49 bytes & Length of ACK and CTS & 29 bytes \\
\hline Header transfer time, $H$ & $121 \mu \mathrm{s}$ & ACK transfer time, $t_{A C K}$ & $106 \mu \mathrm{s}$ \\
\hline RTS length & 35 bytes & RTS transfer time, $t_{R T S}$ & $111 \mu \mathrm{s}$ \\
\hline SIFS & $10 \mu \mathrm{s}$ & DIFS & $50 \mu \mathrm{s}$ \\
\hline EIFS & $212 \mu \mathrm{s}$ & $V$ & $11 \mathrm{Mbps}$ \\
\hline Short retry limit, $N_{s}$ & 7 & Long retry limit, $N_{\ell}$ & 4 \\
\hline Minimal contention window, $W_{0}$ & 32 & Maximal contention window, $W_{m}$ & 1024 \\
\hline
\end{tabular}
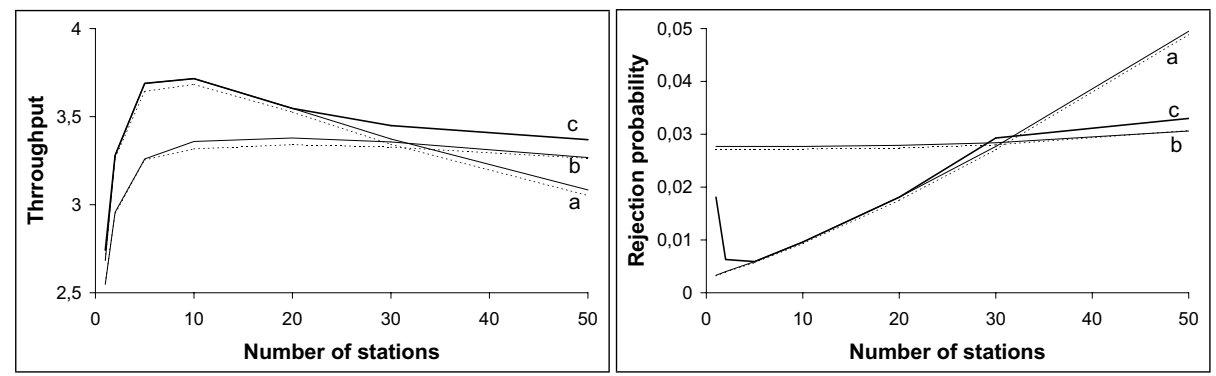

Fig. 3. Throughput (Mbps) and rejection probability versus number of station with $\mathrm{BER}=5 \cdot 10^{-5}$ for (a) the Basic Access mechanism, (b) the RTS/CTS mechanism, and (c) the optimal hybrid mechanism

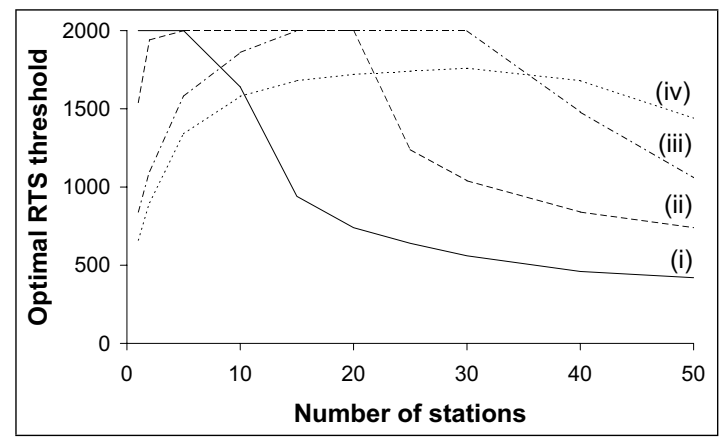

Fig. 4. Optimal RTS threshold (bytes) versus number of station with (i) $\mathrm{BER}=1 \cdot 10^{-5}$, (ii) $\mathrm{BER}=5 \cdot 10^{-5}$, (iii) $\mathrm{BER}=1 \cdot 10^{-4}$, and (iv) $\mathrm{BER}=1.4 \cdot 10^{-4}$

In Figure 3, we present some results of studying the throughput and the averaged rejection probability for the Basic Access and RTS/CTS mechanisms (where $\bar{P}>l_{\max }$ and $\bar{P}=0$, respectively) with varying the number $N$ of stations. Here dotted curves have been obtained by simulation, while our method has been adopted to obtain other curves. First of all, let us note a high accuracy of the analytical model: the errors never exceed $2 \%$ with throughput estimation and $5 \%$ with rejection probability estimation. 
Further, as we could expect, the Basic Access mechanism provides the highest throughput when a number $N$ of stations is small $(N<30$ in Figure 3), while the RTS/CTS mechanism is better when $N$ is large and provides nearly the same throughput with increasing the number of stations.

The bold curves in Figure 3 have been obtained for the hybrid mechanism with the optimal RTS threshold $\bar{P}_{\text {opt }}$ providing the maximal throughput and depending on $N$. The optimizing curves are shown in Figure 4 for various values of BER and have been determined with our analytical method. (A high calculation speed of our method has allowed us to use the exhaustive search of the optimal threshold.) With a low BER (curve (i)), $\bar{P}_{\text {opt }}$ is quite small for large $N$, increases monotonically with decrease of $N$ until some threshold $N_{b}$ (where $\bar{P}_{\text {opt }}$ becomes equal to $l_{\max }+1=2000$ bytes), and remains constant with $N \leq N_{b}$, that is, the Basic Access mechanism is the best for small $N$. With a high BER (curves (ii)-(iv)), a curve $\bar{P}_{\text {opt }}(N)$ is not monotonic, that is, an additional threshold $N_{b}^{0}$ appears somewhere below $N_{b}$ and $\bar{P}_{\text {opt }}$ decreases with decrease of $N$ from $N_{b}^{0}$ to 1 . For example, $N_{b}=30$ and $N_{b}^{0}=15$ with $\mathrm{BER}=1 \cdot 10^{-4}$. Both thresholds increase with BER growth, but $N_{b}^{0}$ increases faster so that the interval, where the Basic Access mechanism is the best, disappears and these thresholds unite into one with a very high BER (see curve (iv)).

Thus, we have obtained the following surprising fact: when stations are few and a BER is high, the best mechanism is not the Basic Access one, but some hybrid mechanism, and the throughput improvement achieved by this optimization is significant. For example, with $N=2$ and $\mathrm{BER}=1 \cdot 10^{-4}, S=1.44 \mathrm{Mbps}$ for the Basic Access mechanism and $S=1.62 \mathrm{Mbps}$ for the optimal hybrid mechanism with $\bar{P}=\bar{P}_{\text {opt }}=1100$ bytes.

This case of few stations in a LAN can seem "exotic" and negligible, but keeping in mind that we considered only active stations, it corresponds to a reallife situation of low traffic. As Figure 3 shows, the throughput improvement in the considered case is achieved at the expense of worsening a rejection probability: in the above example, $\bar{p}_{r e j}=0.057$ for the Basic Access mechanism and $\bar{p}_{r e j}=$ 0.131 for the optimal hybrid mechanism. It can be explained in the following way. When stations are few and a BER is high, a collision probability is small and a failure probability is equal approximately to a noise-induced distortion probability for a DATA frame. So we can assume that a maximal number of attempts of transmitting a packet is equal to $N_{d}=4$ if the packet is transmitted by the RTS/CTS mechanism and to $N_{s}=7$ with the Basic Access mechanism. For a given packet and BER, the less maximal number of attempts, the larger the rejection probability, the less the mean value of backoff intervals anticipating transmission attempts, and hence the larger the throughput.

\section{Conclusions}

In this paper, a continuation of [5]-[7], a simple analytical method is developed for estimating the throughput of a wireless LAN controlled by the DCF scheme of IEEE 802.11 protocol and operating under saturation and in noise. Besides the 
throughput, the probability of a packet transfer rejection due to the attainment of the limiting values specified by the Standard [1] for the number of attempts for transferring long and short frames is evaluated. According to numerical results, our method is quite exact and can be considered as an effective tool for both investigating the influence of bit error rate on the wireless LAN performance indices and tuning optimally the protocol parameters.

Extensions of the developed method to take into account of a possible presence of hidden stations as well as to consider the real-life situations when traffic generated by wireless LAN stations is non-uniform and non-saturating seem possible and are proposed as a future research activity. In order to tackle new research issues generated by the use of wireless LANs as Internet access networks, we plan also to apply the results of studying the 802.11 MAC layer for investigating the interaction between this protocol and the TCP/IP protocol stack (i.e., the protocols of Internet).

\section{References}

1. Wireless LAN Medium Access Control (MAC) and Physical Layer (PHY) Specifications. ANSI/IEEE Std 802.11, 1999 Edition.

2. Weinmiller, J., Schlager, M., Festag, A., et al.: Performance Study of Access Control in Wireless LANs - IEEE 802.11 DFWMAC and ETSI RES 10 HYPERLAN. Mobile Networks and Applications 2 (1997) 55-76

3. Chhaya, H.S. and Gupta, S.: Performance Modeling of Asynchronous Data Transfer Methods of IEEE 802.11 MAC Protocol. Wireless Networks 3 (1997) 217-234

4. Ho, T.S. and Chen, K.C.: Performance Analysis of IEEE 802.11 CSMA/CA Medium Access Control Protocol. Proc. 7th IEEE Int. Symp. on Personal, Indoor and Mobile Radio Communications (PIMRC'96), Taipei, Taiwan (1996) 407-411

5. Bianchi, G.: Performance Analysis of the IEEE 802.11 Distributed Coordination Function. IEEE Journal on Selected Areas in Communications 18 (2000) 535-548

6. Calí, F., Conti, M., and Gregory, E.: Dynamic Tuning of the IEEE 802.11 Protocol to Achieve a Theoretical Throughput Limit. IEEE/ACM Transactions on Networking 8 (2000) 785-799

7. Vishnevsky, V.M. and Lyakhov, A.I.: IEEE 802.11 Wireless LAN: Saturation Throughput Analysis with Seizing Effect Consideration. Cluster Computing 5 (2002) 133-144

8. T.J. Schriber: Simulation using GPSS. John Wiley \& Sons (1974)

9. Higher-Speed Physical Layer Extension in the $2.4 \mathrm{GHz}$ Band. Supplement to [1] 